\title{
Comparison of patient-controlled epidural analgesia with patient-controlled intravenous analgesia for laparoscopic radical prostatectomy
}

\author{
Department of Anesthesia and Pain Medicine, Biomedical Research Institute, \\ Pusan National University Hospital, Busan, Korea \\ Boo Young Hwang, Jae Young Kwon, So Eun Jeon, Eun Soo Kim, \\ Hyae Jin Kim, Hyeon Jeong Lee, and Jihye An
}

Background: Patient-controlled epidural analgesia (PCEA) is known to provide good postoperative analgesia in many types of surgery including laparoscopic surgery. However, no study has compared PCEA with patient-controlled intravascular analgesia (PCIA) in laparoscopic radical prostatectomy (LARP). In this study, the efficacy and side effects of PCEA and PCIA after LARP were compared.

Methods: Forty patients undergoing LARP were randomly divided into two groups: 1) a PCEA group, treated with $0.2 \%$ ropivacaine $3 \mathrm{ml}$ and $0.1 \mathrm{mg}$ morphine in the bolus; and 2) a PCIA group, treated with oxycodone $1 \mathrm{mg}$ and nefopam $1 \mathrm{mg}$ in the bolus. After the operation, a blinded observer assessed estimated blood loss (EBL), added a dose of rocuronium, performed transfusion, and added analgesics. The numeric rating scale (NRS), infused PCA dose, and side effects were assessed at 1, 6, 24, and $48 \mathrm{~h}$.

Results: EBL, added rocuronium, and added analgesics in the PCEA group were less than those in the PCIA group. There were no significant differences in side-effects after the operation between the two groups. Patients were more satisfied with PCEA than with PCIA. The NRS and accumulated PCA count were lower in PCEA group.

Conclusions: Combined thoracic epidural anesthesia could induce less blood loss during operations. PCEA showed better postoperative analgesia and greater patient satisfaction than PCIA. Thus, PCEA may be a more useful analgesic method than PICA after LARP. (Korean J Pain 2018; 31: 191-8)

Key Words: Epidural injection; Intravenous administration; Laparoscopes; Morphine; Oxycodone; Pain measurement; Patient-controlled analgesia; Postoperative pain; Prostatectomy; Ropivacaine; Thoracic vertebrae.

Received March 13, 2018. Revised June 20, 2018. Accepted June 20, 2018.

Correspondence to: Jae Young Kwon

Department of Anesthesia and Pain Medicine, Biomedical Research Institute, Pusan National University Hospital, 179 Gudeok-ro, Seo-gu, Busan 49241, Korea

Tel: +82-51-240-7399, Fax: +82-51-242-7466, E-mail: jykwon@pusan.ac.kr

(c) This is an open-access article distributed under the terms of the Creative Commons Attribution Non-Commercial License (http:// creativecommons.org/licenses/by-nc/4.0/), which permits unrestricted non-commercial use, distribution, and reproduction in any medium, provided the original work is properly cited.

Copyright (c) The Korean Pain Society, 2018 


\section{INTRODUCTION}

Prostate cancer is the most common cancer in men. Its incidence has increased annually [1]. Laparoscopic radical prostatectomy (LARP) is one of the effective treatments for prostate cancer. Laparoscopic surgery is known to bring decreased postoperative pain and early recovery. However, many patients often complain of post-incisional pain that causes postoperative atelectasis, agitation, cardiovascular instability, and prolonged hospitalization [2-4].

Patient-controlled analgesia (PCA) is usually used for postoperative pain management $[5,6]$. However, there is a lack of evidence to develop an optimal pain management protocol in patients undergoing LARP. Patient-controlled epidural analgesia (PCEA), patient-controlled intravascular analgesia (PCIA), and single use analgesics have been applied for postoperative pain management in LARP [7-9]. Although PCEA is known to be more advantageous in controlling pain than PCIA, PCEA is a pain-inducing procedure that is inconvenient due to the requirement that the patient remain catheterized on the back. PCIA also has side effects from the use of opioids.

Therefore, it is necessary to investigate which analgesic method is more advantageous for LARP. The objective of this study was to investigate the analgesic effects of PCEA and PCIA on acute postoperative pain in LARP.

\section{MATERIALS AND METHDOS}

\section{Study design}

A prospective and randomized study was conducted at a university hospital between July 2016 and January 2017. This study was approved by the Institutional Review Board for Human Experiments at University Hospital Medical Research Institute. It was registered with the Clinical Research Information Service, conforming to the World Health Organization International Clinical Trials Registry Platform (WHO-ICTRP) (registration number: KCTOOO 2245). All patients provided informed consent before surgery.

\section{Subjects}

Forty ASA I or II patients aged 45 to 75 years old who were scheduled for LARP were included. Patients who did not qualify for treatment with morphine, nefopam, ropivacaine, oxycodone, or epidural procedures, who could not use the numeric rating scale (NRS), or who had a psychological disorder, chronic pain disorder, renal failure, hepatic failure, or preoperative administration of medications including opioids, antidepressants, gabapentin, pregabalin, and carbamazepine, were excluded. Patients were randomly divided into a PCEA group and a PCIA group. We explained both methods for pain management to all patients. If they refused either method, they were excluded.

\section{Treatment}

After patients arrived in the operating room, their heart rate (HR), systolic blood pressure (SBP), diastolic blood pressure (DBP), mean blood pressure (MBP), and respiratory rate (RR) were obtained. Glycopyrrolate $(0.2 \mathrm{mg})$ was intramuscularly injected in all patients $30 \mathrm{~m}$ before induction of anesthesia. The bispectral index (BIS, XP version 4.1; Aspect Medical Systems, Newton, MA) was monitored to measure the depth of the anesthesia. Train of four (TOF) monitoring was used to measure the depth of the neuromuscular block. The patient's left arm was spread with the palm facing up and two electrodes were placed over the path of the ulnar nerve. The distal electrode was placed at the flexor crease and the second electrode was placed 1-2 $\mathrm{cm}$ proximal to the first, parallel to the flexor carpi ulnaris tendon.

The epidural catheter was inserted before anesthesia induction in the PCEA group. With the patient in the right side lateral position, the catheter was inserted to a depth of $3-4 \mathrm{~cm}$ on the $10^{\text {th }}$ thoracic vertebra level (T10). We used a Touhy's needle for the procedure, and a glass syringe filled with normal saline used in loss of resistance technique. After the test dose (3 $\mathrm{ml}$ of $1 \%$ lidocaine and $1: 200,000$ epinephrine) was administered, patients received $0.2 \%$ ropivacaine $7 \mathrm{ml}$ and $3 \mathrm{mg}$ morphine sulfate as a loading dose before the incision.

Anesthesia was induced with $2 \mathrm{mg} / \mathrm{kg}$ propofol and 0.8 $\mathrm{mg} / \mathrm{kg}$ rocuronium. Desflurane at a concentration of 1 minimal alveolar concentration (MAC) was used to maintain anesthesia in both groups. Its dose was increased or decreased within $20 \%$ depending on vital sign and BIS. Tidal volume of $7 \mathrm{ml} / \mathrm{kg}$ was used based on ideal body weight. 
Respiratory rate was controlled to maintain end-tidal $\mathrm{CO}_{2}$ between 35 and $40 \mathrm{mmHg}$.

Remifentanil in the PCIA group and the bolus dose ( $0.2 \%$ ropivacaine $3 \mathrm{ml}$ and $0.1 \mathrm{mg}$ morphine sulfate) in the PCEA group were controlled to maintain vital signs within $20 \%$ of their basal levels. In the PCEA group, the lock-out interval was 30 minutes. There was no preset four-hour limit volume. If TOF count was above 2 or a surgeon requested relaxation for a better surgical view, 0.1 $\mathrm{mg} / \mathrm{kg}$ rocuronium was injected. An antiemetic and loading dose of $20 \mathrm{mg}$ nefopam and $5 \mathrm{mg}$ oxycodone in the PCIA group, or $0.2 \%$ ropivacaine $3 \mathrm{ml}$ and $0.1 \mathrm{mg}$ morphine sulfate in the PCEA group were administered at 30 minutes before the end of surgery. Patients received pain control via a PCA device (GemStar ${ }^{\circledR}$ Infusion System, Hospira, Lake Forest, IL) in both groups. PCIA was started after the end of surgery with a bolus dose of $1 \mathrm{ml}$ (oxycodone $1 \mathrm{mg}$ and nefopam $1 \mathrm{mg}$ ), a lock-out interval of 6 minutes, and a four-hour limit volume of $40 \mathrm{ml}$. In this study, both PCIA and PCEA were set at bolus only mode.

After the operation, $10 \mathrm{mg}$ pyridostigmine and $0.4 \mathrm{mg}$ glycopyrrolate were administered intravenously to all patients. They were then transferred to the post-anesthesia care unit (PACU). They stayed there until the Aldrete score was greater than 8 . If patients complained of severe pain (NRS > 5), we recommended that they press the button instead of administering rescue analgesics. If the pain was not relieved, fentanyl $50 \mu \mathrm{g}$ was administered.

\section{Assessment}

The primary outcome was considered to be the analgesic effect, while secondary outcomes included the satisfaction of patients, incidence of side-effects, and anesthetic outcomes such as the estimated blood loss (EBL), vital sign, BIS, TOF, added rocuronium count, and so on.

Before the operation, history of motion sickness and smoking history were checked. We recorded laparoscopic gas insufflation time, gas insufflated pressure, peak airway pressure (Ppeak), end-tidal $\mathrm{CO}_{2}\left(\mathrm{ETCO}_{2}\right), \mathrm{RR}$, vital signs, BIS score, TOF count, desflurane concentration, added rocuronium count (0.1 mg/kg per bolus), and use of ephedrine during the operation.

NRS, accumulative PCA count, side effects, Ramsay sedation scale ( 1 = anxious, agitated, or restless; $2=$ co- $^{-}$ operative, oriented, and tranquil; 3 = responds to com- mand; 4 = brisk response to a light glabellar tap or loud auditory stimulus; 5 = sluggish response to a light glabellar tap or loud auditory stimulus; and $6=$ no response to the stimuli), and adjuvant drugs were recorded at 1, 6, 24, 48 $\mathrm{h}$ after the operation. Accumulated PCA count recorded the actual number of times that a bolus was administered, not the number of times it was pressed.

Nausea was classified into three grades: $1=$ mild; 2 $=$ moderate; and $3=$ severe. If patients complained of nau sea above grade 2 , then $4 \mathrm{mg}$ ondansetron was intravenous administered. Vomiting was graded into two grades: $1=<4$ times of vomiting; and $2=\geq 4$ times of vomiting. The satisfaction levels of the patients were obtained at 48 $\mathrm{h}$ after the operation. Patients were asked to rank their satisfaction with their pain management, side-effects, and inconvenience according to the following scale: $1=$ very unsatisfactory; 2 = unsatisfactory; 3 = neutral; 4 = sat isfactory; and $5=$ very satisfactory.

\section{Statistical analysis}

An estimated sample size indicated that 16 patients per group would give a $\beta$-risk of $80 \%$ at $\alpha$-level of 0.05 for detecting a difference in NRS (2 vs. 3) of at least 1 at 1 hour after the operation with a standard deviation of 1.0 for each group in the preliminary test. We enrolled 40 subjects for this study, to be prepared for a $10 \%$ exclusion rate.

Demographic data are expressed as mean \pm SD. They were compared using the Student's t-test and Chi-square test. Anesthetic data of the operation were compared using the Mann-Whitney test. The incidence of side effects was compared using the Chi-square test. Satisfaction scores were also compared using the Chi-square test. Laparoscopic gas insufflation pressure, Ppeak, $\mathrm{ETCO}_{2}, \mathrm{RR}$, vital signs, BIS score, TOF, NRS, and accumulated PCA dose were compared using two-way repeated measures ANOVA.

A probability of $<0.05$ was considered to be statistically significant. SPSS version 21.0 (IBM statistics data editor SPSS Inc., Chicago, IL) was used for all statistical analyses.

\section{RESULTS}

Two patients were excluded due to refusal to participate 
CONSORT 2010 Flow Diagram

Enrollment
Assessed for eligibility $(n=40)$
Excluded $(n=2)$

- Not meeting inclusion criteria $(n=0)$

- Declined to participate $(n=2)$

- Other reasons $(n=0)$

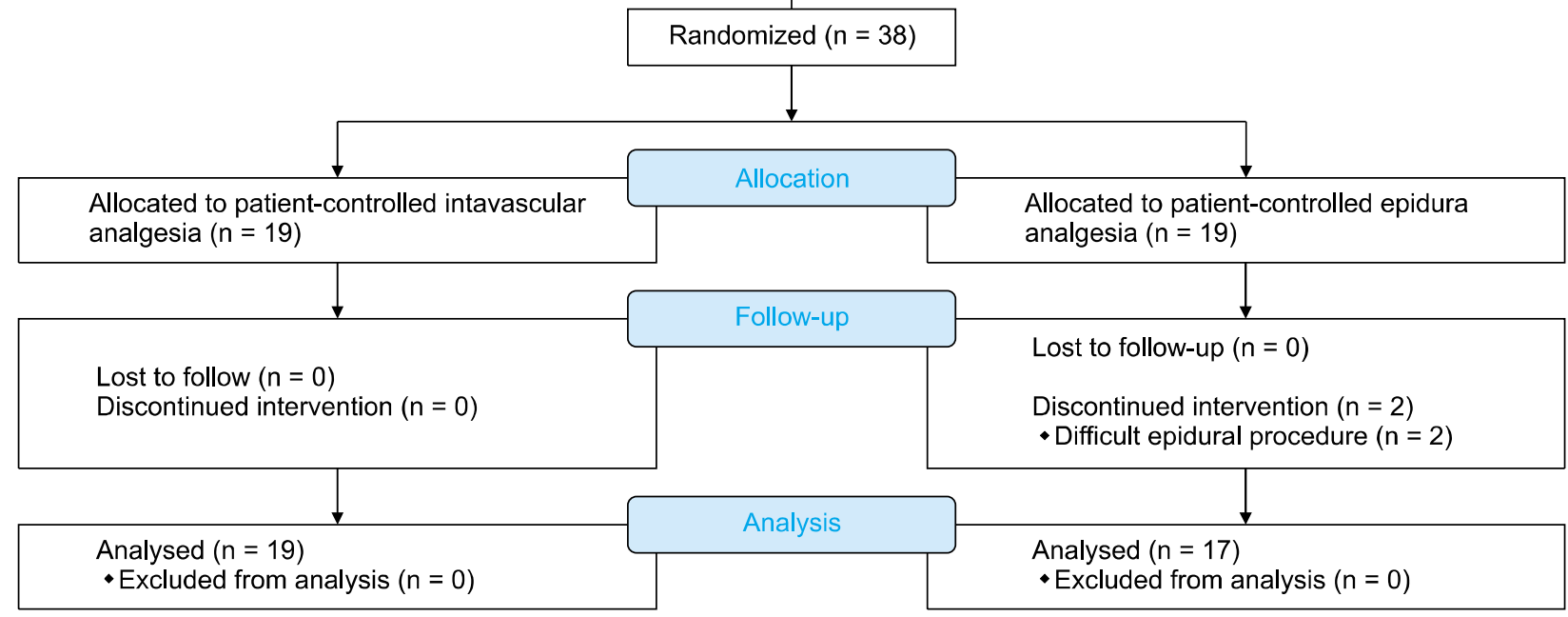

Fig. 1. Study flow chart with individual causes of study interruptions and dropouts. The flow chart of this study was according to the CONSORT Statement.

Table 1. Demographic Data

\begin{tabular}{lccc}
\hline & PCIA & PCEA & $P$ value \\
\hline Weight $(\mathrm{kg})$ & $68.2(12.8)$ & $68.3(7.3)$ & 0.08 \\
Height $(\mathrm{cm})$ & $167.0(7.9)$ & $168.7(5.5)$ & 0.44 \\
$\quad$ ABW & $65.2(9.0)$ & $67.9(6.9)$ & 0.34 \\
Age (yrs.) & $65.3(11.9)$ & $66.8(5.6)$ & 0.44 \\
History of motion & 1 & 3 & 0.24 \\
$\quad$ sickness or PONV (n) & & & \\
Smoking history (n) & 2 & 0 & 0.169 \\
\hline
\end{tabular}

There are no differences in weight, height, adjustable body weight (ABW), history of motion sickness and smoking, and age between both groups. Data are expressed as mean \pm standard deviation (SD).

in this study while two patients in the PCEA group discontinued participation because of difficult condition for the epidural procedure (Fig. 1).

There were no significant differences in weight, height, adjustable body weight (ABW), age, anesthesia time, operation time, gas insufflation time, insufflated pressure, infused fluid, use of ephedrine, history of motion sickness, smoking, incidence of transfusion, or hospital stay be- tween the two groups. EBL, added rocuronium count, and additional analgesics count in the PCEA group were less than those in the PCIA group (Table 1, 2, all $P<0.05$ ). There were no significant differences in side-effects after the operation between the two groups (Table 3).

Patients were more satisfied with PCEA than with PCIA $(P=0.049)$.

Ppeak, $\mathrm{RR}, \mathrm{ETCO}_{2}, \mathrm{HR}$, saturation, and TOF count were not significantly different between the two groups. $\mathrm{SBP}$, DBP, and MBP in the PCEA group were lower than those in the PCIA group (Fig. 2, all $P<0.005$ ).

The NRS and accumulated PCA count were lower in the PCEA group than those in the PCIA group (Fig. 3, both $P<0.005)$.

\section{DISCUSSION}

In this study, we showed that thoracic epidural anesthesia was more effective by producing less estimated blood loss, and that patients in the PCEA group showed lower NRS scores than those in the PCIA group. PCEA provided better pain management and greater satisfaction to patients than 
Table 2. Anesthetic Data

\begin{tabular}{lccc}
\hline & PCIA & PCEA & $P$ value \\
\hline Anesthesia time (min) & $154.7(35.0)$ & $146.5(21.6)$ & 0.14 \\
Operation time (min) & $129.2(33.3)$ & $119.7(19.8)$ & 0.17 \\
Gas insufflation time (min) & $102.1(27.3)$ & $92.8(17.9)$ & 1.06 \\
Gas insufflated pressure (mmHg) & $11.9(1.8)$ & $12.4(1.6)$ & 0.99 \\
Fluid & & & 0.48 \\
Crystalloid (ml) & $1242.1(557.1)$ & $1074.1(552.9)$ & 0.82 \\
Colloid (ml) & $52.6(157.6)$ & $58.8(166.1)$ & $0.04^{*}$ \\
Estimated Blood loss (ml) & $392.1(123.9)$ & $288.2(140.9)$ & 0.18 \\
Use of Ephedrine (n) & $0.74(1.1)$ & $1(2.0)$ & $0.014^{*}$ \\
Added rocuronium (n) & $1.63(1.6)$ & $1.06(0.8)$ & 0.487 \\
Surgeon's request for relaxation (n) & 2 & 0 & - \\
Postoperative transfusion (ml) & 0 & $0.06(0.2)$ & $0.016^{*}$ \\
Added analgesics (n) & $0.68(1.9)$ & $5.29(1.5)$ & 0.565 \\
Duration of hospital stay (day) & $5.16(1.7)$ & &
\end{tabular}

There are no differences in anesthesia time, operation time, gas insufflation time, insufflated pressure, infused fluid, use of ephedrine, incidence of transfusion and duration of hospital stay between both groups. Estimated blood loss and added rocuronium count $(0.2 \mathrm{mg} / \mathrm{kg}$ per 1 count) and analgesics count in the patient-controlled epidural analgesia (PCEA) group are less than those of the patient-controlled intravascular analgesia (PCIA) groups. Data are expressed as mean and standard deviation $(S D) .{ }^{*}: P<0.05$ between groups.

Table 3. Side Effects of Patient-Controlled Analgesia

\begin{tabular}{|c|c|c|c|c|c|c|}
\hline & & & $\mathrm{PCIA}$ & PCEA & Total & $P$ value \\
\hline \multirow[t]{8}{*}{ Nausea } & $<1$ hour & 1 & 1 & 2 & 3 & 1.261 \\
\hline & & 2 & 3 & 1 & 4 & \\
\hline & $<6$ hours & 1 & 0 & 1 & 2 & 0.176 \\
\hline & & 2 & 4 & 0 & 6 & \\
\hline & & 3 & 1 & 1 & 5 & \\
\hline & 6-24 hours & 2 & 1 & 0 & 1 & 0.388 \\
\hline & & 3 & 1 & 0 & 1 & \\
\hline & $<48$ hours & 2 & 1 & 0 & 1 & 0.337 \\
\hline Vomiting & $<6$ hours & 1 & 0 & 1 & 1 & 0.284 \\
\hline Headache & & & 0 & 0 & 0 & - \\
\hline Shivering & $<6$ hours & & 0 & 2 & 2 & 0.124 \\
\hline Pruritus & $<6$ hours & & 0 & 1 & 1 & 0.284 \\
\hline Delirium & & & 0 & 0 & 0 & - \\
\hline $\begin{array}{c}\text { Respiratory } \\
\text { depression }\end{array}$ & & & 0 & 0 & 0 & - \\
\hline $\begin{array}{l}\text { Problems } \\
\text { related } \\
\text { to epidural } \\
\text { procedure }\end{array}$ & & & 0 & 0 & 0 & - \\
\hline
\end{tabular}

There are no significant differences in side effects after the operation between both groups. Data are expressed as numbers. Nausea is classified into three grades: $1=$ mild; 2 = moderate; and 3 = severe. Vomiting is classified into two grades: $1=<$ 4 times of vomiting; $2=\geq 4$ times of vomiting.
PCIA.

We used oxycodone and nefopam for PCIA and morphine and ropivacaine for PCEA, based on our hospital's protocol. We decided that it was unnecessary to unify all drugs because patients adjust the dose by using PCA. Since PCA itself does not accurately determine the dose and it is a patient-controlled dose, it can be concluded that the efficacy is good if the NRS scores were kept low. In addition, even if efficacy is good, if PCA is accompanied by many side effects, it may be difficult to apply it to the actual patient. In the present study, it was not accompanied by many side effects.

The primary outcome in this study was the analgesic effects on acute postoperative pain. Sample size was determined by detecting difference in the NRS at 1 hour postoperatively. Usually, postoperative pain is more severe in the acute postoperative period and one hour is sufficient to eliminate the effects of opioids and anesthetics administered during the operation.

There have been many studies about pain management for radical prostatectomy. They usually show that PCEA is an efficient method for pain management after radical prostatectomy [9]. Some studies have suggested that the disadvantages of PCEA appear to outweigh its advantages. Longer hospital stays and higher rates of epidural-related 

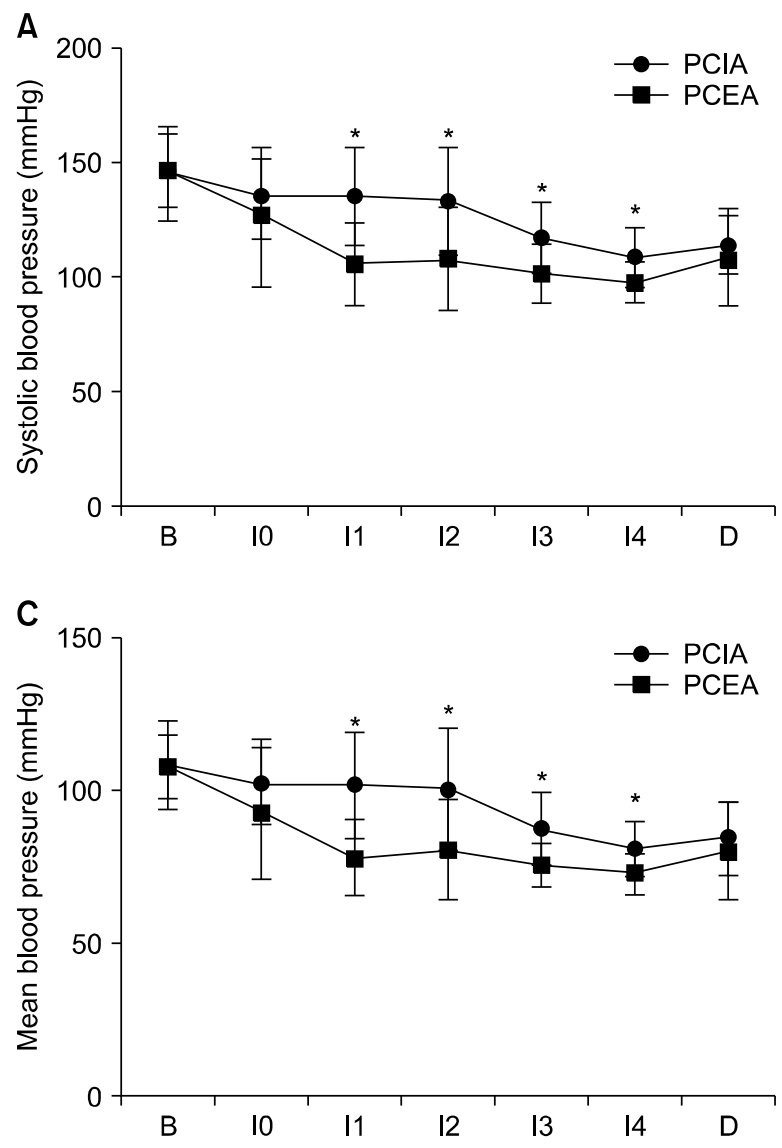

side-effects have been found in the epidural group (17/115 $=14.8 \%)$ than those in the non-epidural group $(6 / 124=$ 4.8\%) [10]. Wheatley et al. [11] have suggested that, although minimally invasive procedures are less painful, there are more adverse effects associated with epidural analgesia. However, in this study, hospital stays in the PCEA group were not significantly different from those in the PCIA group. Epidural related side-effects did not show up either. If there were more epidural procedure-related complications in the PCEA group, the conclusion might have been different. Patients in the PCEA group needed fewer analgesics, and pain complaints were fewer than with patients in the PCIA group after the operation, without showing increased adverse effects related to epidural analgesia. These results suggest that less pain, less analgesic need, and less opioid consumption in the PCEA group might have led to more satisfied patients.

Formulations of PCIA and PCEA are determined by a hospital's protocol. Both PCIA and PCEA are set with bolus only mode because there is no difference in pain management between bolus with background infusion and bolus

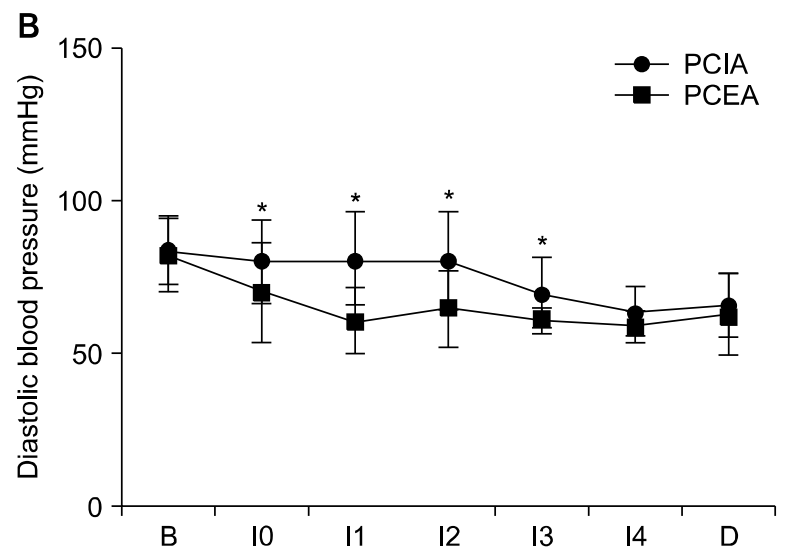

Fig. 2. Blood pressure during laparoscopic radical prostatectomy. 2a. Systolic blood pressure (SBP), 2b. Diastolic blood pressure (DBP), 2c. Mean blood pressure (MBP). Systolic blood pressure (SBP), diastolic blood pressure (DBP), and mean blood pressure (MBP) of the PCEA group are lower than those of the PCIA group. B: baseline, 10: intubation time, 11: incision time, 12: insufflation time, 13: prostatectomy time, 14: anastomosis time, D: deflation. ${ }^{\star} P<0.05$ compared with PCIA group.

only groups $[12,13]$. For PCIA, oxycodone and nefopam are used. Nefopam is a centrally-acting non-opioid analgesic which has no effect on bleeding time or platelet aggregation. A nefopam and oxycodone combination shows similar efficacy, but lower incidence of nausea within $6 \mathrm{~h}$ after the operation compared to ketorolac and oxycodone in PCIA after gynecologic surgery [14].

Epidural morphine and ropivacaine were used in this study. Compared to local anesthetic or an opioid alone, a local anesthetic-opioid combination provides superior postoperative analgesia. It limits regression of the sensory blockade and possibly decreases the dose of the local anesthetic administered [13]. A morphine and ropivacaine combination has been found to be superior to the fentanyl and ropivacaine combination in thoracic surgery [15]. There was a reduced number of infusion interventions and less inadequate patient analgesia [15]. Another study has also shown that morphine and ropivacaine PCEA demonstrates an excellent safety and efficacy profile compared to sufentanil and ropivacaine PCEA [16].

In the present study, combined thoracic epidural anes- 
A

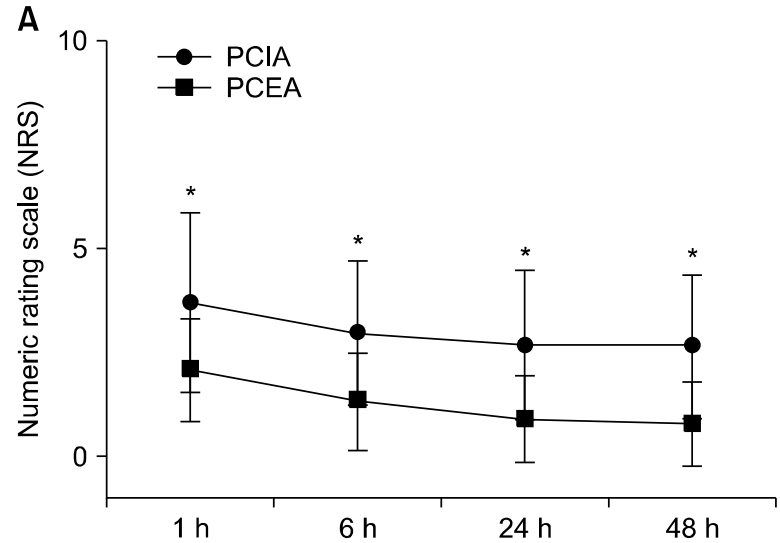

B

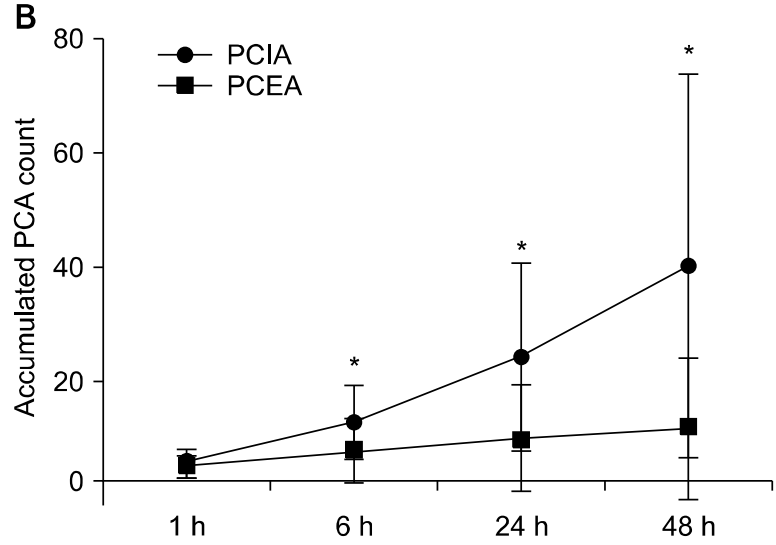

Fig. 3. Numeric rating scale (NRS) of pain intensity and accumulated PCA count at 1, 6, 24 and $48 \mathrm{~h}$ after the operation. (A) Numeric rating scale (NRS) of pain intensity at 1, 6, 24 and $48 \mathrm{~h}$ after the operation. Both groups show a decrease in pain as the time passed. In all period, lower NRS are recorded in Patient-controlled epidural analgesia (PCEA) group than patient-controlled intravascular analgesia (PCIA). Values are expressed as mean $\pm \mathrm{SD}$. ${ }^{\star} P<0.05$ compared with PCIA group. (B) Accumulated PCA count at 1, 6, 24 and $48 \mathrm{~h}$ after the operation. Lower accumulated patient-controlled analgesia (PCA) counts are recorded in the patient-controlled epidural analgesia (PCEA) group than the patient-controlled intravascular analgesia (PCIA). Values are expressed as mean \pm SD. ed with PCIA group. Data are expressed as mean \pm SD.

thesia did not improve surgical field or respiratory conditions during the operation. There was no significant difference in Ppeak or intraabdominal gas insufflation pressure either. One study has suggested that combined general and epidural anesthesia may attenuate the severity of postoperative diaphragmatic dysfunction after robot-assisted laparoscopic radical prostatectomy compared to conventional general anesthesia [7]. Diaphragmatic inspiratory amplitude was not measured in this study. The use of rocuronium in the PCEA group was decreased, suggesting that epidural anesthesia could help muscle relaxation for surgery to maintain an optimal surgical condition. Significant reduction in the requirement of vecuronium during maintenance in the PCEA group has also been observed in another study [17].

Our findings revealed that estimated blood loss during operation in the PCEA group was lower than that in the PCIA group. Decreased blood pressure from epidural anesthesia might have influenced blood loss. Hendolin et al. [18] have also shown that the mean perioperative blood loss during operations under epidural analgesia is significantly less than that under general anesthesia $(370 \pm 34 \mathrm{ml}$ vs. $590 \pm 34 \mathrm{ml}$ vs. $590 \pm 35 \mathrm{ml}$ ). However, another study has reported that the hemoglobin difference between preand the first postoperative day did not show group differences in overall blood loss (general anesthesia + thoracic epidural analgesia: $665 \pm 431.5 \mathrm{ml}$; only general anesthesia: $705 \pm 881 \mathrm{ml} ; P=0.73)$ or transfusion rate $(0.4 \%$ intraoperatively; 2.55\% postoperatively; $P=1.0$ ) [19].

In conclusion, PCEA showed a greater effect on postoperative pain relief and patient satisfaction than PCIA. Epidural anesthesia also induced less blood loss during the operation. Although PCEA has some risk of procedure-related side effects with less benefit on minimal invasive operation, it is an effective pain controlling method for LARP.

\section{ACKNOWLEDGEMENTS}

This work was supported by a 2-year Research Grant from Pusan National University.

\section{REFERENCES}

1. Siegel R, Ward E, Brawley O, Jemal A. Cancer statistics, 2011: the impact of eliminating socioeconomic and racial disparities on premature cancer deaths. CA Cancer J Clin 2011; 61: 212-36.

2. Laufenberg-Feldmann R, Kappis B, Mauff S, Schmidtmann I, Ferner M. Prevalence of pain 6 months after surgery: a prospective observational study. BMC Anesthesiol 2016; 16: 91.

3. Chen $\mathrm{K}, \mathrm{Xu} X \mathrm{X}$, Zhang RC, Pan Y, Wu D, Mou YP. Systematic review and meta-analysis of laparoscopy-assisted 
and open total gastrectomy for gastric cancer. World J Gastroenterol 2013; 19: 5365-76.

4. Bisgaard T, Klarskov B, Rosenberg J, Kehlet $H$. Characteristics and prediction of early pain after laparoscopic cholecystectomy. Pain 2001; 90: 261-9.

5. Hudcova J, McNicol E, Quah C, Lau J, Carr DB. Patient controlled opioid analgesia versus conventional opioid analgesia for postoperative pain. Cochrane Database Syst Rev 2006: CD003348.

6. Capdevila X, Moulard S, Plasse C, Peshaud JL, Molinari N, Dadure C, et al. Effectiveness of epidural analgesia, continuous surgical site analgesia, and patient-controlled analgesic morphine for postoperative pain management and hyperalgesia, rehabilitation, and health-related quality of life after open nephrectomy: a prospective, randomized, controlled study. Anesth Analg 2017; 124: 336-45.

7. Oh YJ, Lee JR, Choi YS, Koh SO, Na S. Randomized controlled comparison of combined general and epidural anesthesia versus general anesthesia on diaphragmatic function after laparoscopic prostatectomy. Minerva Anestesiol 2013; 79: 1371-80.

8. Webster TM, Herrell SD, Chang SS, Cookson MS, Baumgartner RG, Anderson LW, et al. Robotic assisted laparoscopic radical prostatectomy versus retropubic radical prostatectomy: a prospective assessment of postoperative pain. J Urol 2005; 174: 912-4.

9. Joshi GP, Jaschinski T, Bonnet F, Kehlet H; PROSPECT Collaboration. Optimal pain management for radical prostatectomy surgery: what is the evidence? BMC Anesthesiol 2015; 15: 159.

10. Mir MC, Joseph B, Zhao R, Bolton DM, Gyomber D, Lawrentschuk N. Effectiveness of epidural versus alternate analgesia for pain relief after radical prostatectomy and correlation with biochemical recurrence in men with prostate cancer. Res Rep Urol 2013; 5: 139-45.

11. Wheatley RG, Schug SA, Watson D. Safety and efficacy of postoperative epidural analgesia. $\mathrm{Br} J$ Anaesth 2001; 87 : 47-61.

12. Carvalho B, George RB, Cobb B, McKenzie C, Riley ET. Implementation of programmed intermittent epidural bolus for the maintenance of labor analgesia. Anesth Analg 2016; 123: $965-71$.

13. Doyle E, Robinson D, Morton NS. Comparison of patient-controlled analgesia with and without a background infusion after lower abdominal surgery in children. $\mathrm{Br} J$ Anaesth 1993; 71: 670-3.

14. Hwang BY, Kwon JY, Lee DW, Kim E, Kim TK, Kim HK. A randomized clinical trial of nefopam versus ketorolac combined with oxycodone in patient-controlled analgesia after gynecologic surgery. Int J Med Sci 2015; 12: 644-9.

15. Royse CE, Royse AG, Deelen DA. An audit of morphine versus fentanyl as an adjunct to ropivacaine $0.2 \%$ for high thoracic epidural analgesia. Anaesth Intensive Care 2005; 33: 639-44.

16. Delvecchio L, Bettinelli S, Klersy C, Allegri M, Cavalloro F, Braschi A. Comparing the efficacy and safety of continuous epidural analgesia in abdominal and urological surgery between two opioids with different kinetic properties: morphine and sufentanyl. Minerva Anestesiol 2008; 74: 69-76.

17. Agarwal A, Pandey R, Dhiraaj S, Singh PK, Raza M, Pandey $\mathrm{CK}$, et al. The effect of epidural bupivacaine on induction and maintenance doses of propofol (evaluated by bispectral index) and maintenance doses of tentanyl and vecuronium. Anesth Analg 2004; 99: 1684-8.

18. Hendolin H, Alhava E. Effect of epidural versus general anaesthesia on peroperative blood loss during retropubic prostatectomy. Int Urol Nephrol 1982; 14: 399-405.

19. Baumunk D, Strang CM, Kropf S, Schäfer M, Schrader M, Weikert S, et al. Impact of thoracic epidural analgesia on blood loss in radical retropubic prostatectomy. Urol Int 2014; 93: 193-201. 